\title{
Integration of thermographic data with the 3D object model
}

\author{
by M. Kaczmarek*
}

\author{
*Gdansk Univ. of Technology ,Gdansk, Narutowicza 11/12, 80-233,Poland, mariusz.kaczmarek@eti.pg.gda.pl
}

\begin{abstract}
The aim of the paper is to present new method for merging the 3D model data of the measured object with thermograms. Our technique is based on the combination of visual $3 \mathrm{D}$ imaging technique and thermal imaging technique, which maps the $2 \mathrm{D}$ thermograms on to $3 \mathrm{D}$ anatomical mesh model. The combination of these imaging modalities allows the generation of combined $3 \mathrm{D}$ and thermal data from which thermal signatures can be verified and quantitative analysed.
\end{abstract}

\section{Introduction}

Thermography in medical diagnosis has been used for decades [1]. However, thermography is still viewed as an accessory to conventional clinical examination procedures because it only provides qualitative data. The new technology Active Dynamic Thermography (ADT) with external excitations we develop will greatly enhance the usefulness of thermography for clinicians, as it will provide qualitative and quantitative data concerning the physiological and functional state of tissues [2]. Our experience of use ADT in medicine is mainly connected with burn diagnosis [3], cardiosurgery interventions [4] and thermal mammography. The human body is very complicated device with different/irregular shapes, so it is very difficult to precise mapping of temperature distribution over the body surface. It can be solved by using merging technique with $3 \mathrm{D}$ scanned data [5].There are many 3D scanning techniques either active or passive. One of more suitable techniques is structured light 3D scanning technique [6]. After having motivated our research, we present the configuration of the system we have built. Finally we present some early results showing the process of thermogram and $3 \mathrm{D}$ data merging.

\section{Material and Methods}

Medical Active Dynamic Thermography (ADT) is very sensitive and reliable mean of graphically mapping and display skin surface temperature and time constant parameter. It allows physicians to visualise in colour and quantify temperature changes in skin surface and thermal parameters, blood flow of few millimetres under the surface. Many studies and analyses have deal with the problem of thermal measurements and 3D technique development [6][7].

This paper proposes a comprehensive conceptual model that enables combining 3D scanning procedure with thermal imaging to produce 3D object model with merged thermograms. This is a new approaches based on 3D thermograms in medicine. Using the 3D model data acquired by the 3D scanner it is possible to define spatial data that includes shape and temperature distribution information. Also correlating $2 \mathrm{D}$ thermograms in the whole sequence of ADT procedure is easier and can be performed automatically using 3D point registration algorithms.

\subsection{Equipment}

3D scanner features very fast shape acquisition and high accuracy functionalities. It generates 3D data that can be used to obtain anatomical model of body shape. Accuracy of scanned data is up to $0.5 \mathrm{~mm}$ and high point cloud density (to 650 points $/ \mathrm{mm}^{2}$ ) provides high precise geometry scanning and enabling to archive, visualize or to guide virtual analysis of object by using results of 3D scanning. The use high-in-class detector gives the possibility to visualize the smallest changes and visualize every object surface detail. White light 3D scanning technology, use only white spectrum of light which guarantee to user safety scanning of measuring objects. Also, it is possible to scan object in ULTRA-FAST mode ( 2 seconds of scanning, enables for scanning hard objects like human face, plants etc.). Specifications: Scanning Technology: Structural White Light, Detector resolution: 2Mpx, Measuring field [mm ${ }^{2}$ : $150 \times 200$ - 1200x1600. Thermography equipment consist of uncooled FLIR A320G thermal camera and source of excitation.

\subsection{Images Acquiring and Processing}

To obtain spatial data structured light 3D scanner was used. The camera captures greyscale images of deformed projected stripe patterns. Following that 3D mesh model is created. The modern thermographic IR camera FLIR A320G of thermal resolution $0.08 \mathrm{~K}$ and spatial resolution $320 \times 240$ pixels was used. First the data of static thermography (ST) was collected, followed by the ADT procedure. The cooling excitation by the stream of a cold mixture of $\mathrm{CO}_{2}$ and dry air generated by the cryotherapy device was of the sufficient efficiency for getting the final skin temperature to the ambient temperature (Fig. 1) and then it returns to the state of the thermodynamic balance. In this 
case thermal processes during the natural return to the thermal equilibrium are diagnostically essential as this diagnostic tool is able to combine structural and functional imaging. This approach is an attractive diagnostic option as infrared technology is completely non-invasive and aseptic. A tested tissue can be quantitatively assessed by means of the thermal time constant $\left(\tau_{\mathrm{j}}\right)$ - the exponential function parameter defined in equations (1), (2) for $(x, y)$ pixel at time $t$ :

$$
T(x, y, t)=T_{s}(x, y)+\sum_{j=1}^{m} \Delta T_{j}(x, y) \cdot e^{-\frac{t}{\tau_{j}(x, y)}}
$$

for the natural cooling phase following external heating;

$$
T(x, y, t)=T_{s}(x, y)+\sum_{j=1}^{m} \Delta T_{j}(x, y) \cdot\left(-e^{-\frac{t}{\tau_{j}(x, y)}}\right)
$$

for the re-warming phase following external cooling.

The best possible results can be obtained for the condition when the surface temperature of the heart muscle after forced cooling is equal to the ambient temperature. Then the re-warming process of the muscle mainly depends on the thermal properties of the muscle and on the heating flux coming from the body. Example of such diagnostic procedure for blister growing syndrome is shown on Fig. 1.

a)



b)

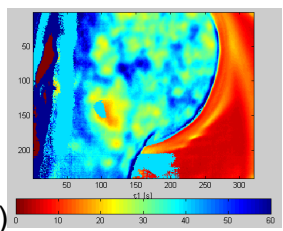

Fig. 1. Examples of medical application - blister forming monitoring, a) photo, b) parametric image of time constant in ADT procedure

3D thermograms allow modelling different processes based on scientific studies of medicine and biomedicine (e.g. breast cancer temperature dissipation, blister forming). This means new data analysis methods have to be invented and new method of visualisation can be applied to enable medical professionals setting up a more reliable and comprehensive diagnosis. The main idea is presented on Fig. 2.

We develop a 3D thermography imaging standardization technique to allow quantitative data analysis. Active Dynamic Thermography with cooling external device is very sensitive and reliable mean of graphically mapping and display skin surface temperature and thermal model parameters as e.g. thermal time constant.

3D meshing
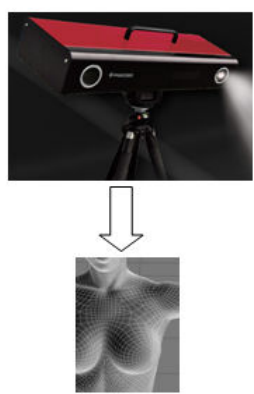

Thermal/ADT Measurement
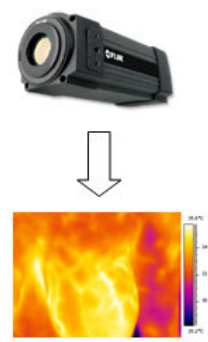

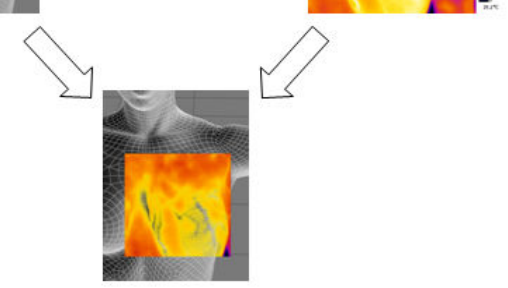

Fig. 2. Merging procedure of $3 D$ model with thermogram - concept

It allows doctors to visualise in colour and quantify temperature changes in skin surface. The spectrum of colours indicates both hot and cold responses. Here, our technique is based on the combination of visual 3D imaging technique and thermal imaging technique, which maps the $2 \mathrm{D}$ thermography images on to $3 \mathrm{D}$ anatomical model. Then we rectify the $3 \mathrm{D}$ thermogram into a view independent thermogram and conform it a standard shape template. The 
combination of these imaging facilities allows the generation of combined $3 \mathrm{D}$ and thermal data from which thermal signatures can be quantified.

Integration of thermographic data with 3D model is shown in more detailed manner on fig.3. Process is started form acquiring the 3D data using optical white light 3D scanner. Following this we obtain the cloud of points and the next step is to reconstruct triangle mesh. Now we have the surface composed of triangle pieces, so we can calculate the surface normal. The normal is often used in computer graphics to determine a surface's orientation toward a light source for flat shading. For a convex polygon (such as a triangle), a surface normal can be calculated as the vector cross product of two (non-parallel) edges of the polygon. The normal to surfaces is used to estimation of power which is emitted from the surface to the ambient according to Lambert's cosine law - the radiant intensity or luminous intensity observed from an ideal diffusely reflecting surface or ideal diffuse radiator is directly proportional to the cosine of the angle $\theta$ between the observer's line of sight and the surface normal - Fig. 3: angle correction procedure. The main diagnostic thermal data are recorded according to ADT procedure [1] and the parametric images of exponential thermal model according to equation (1) or (2) are reconstructed. Next basing on prepared sets of data the registration are done. Registration is necessary in order to be able to integrate the data obtained from these different measurements (optical $3 \mathrm{D}$ and thermal 2D). We still use traditional technique for performing manual image registration, in which an operator chooses corresponding control points (CP) in 3D model and thermal or parametric images. Applied transformation models are linear transformations, which include rotation, scaling, translation, and other affine transforms.[8] Linear transformations are global in nature, so if you want to perform local geometric transformations it should be used so called 'elastic' or 'nonrigid' transformations. These transformations are capable of locally warping the target image to align with the reference image.

Finally, the data merging/integration algorithm is used with bilinear interpolation calculation for a new pixels of thermal texture and Lambert law correction for ST images.

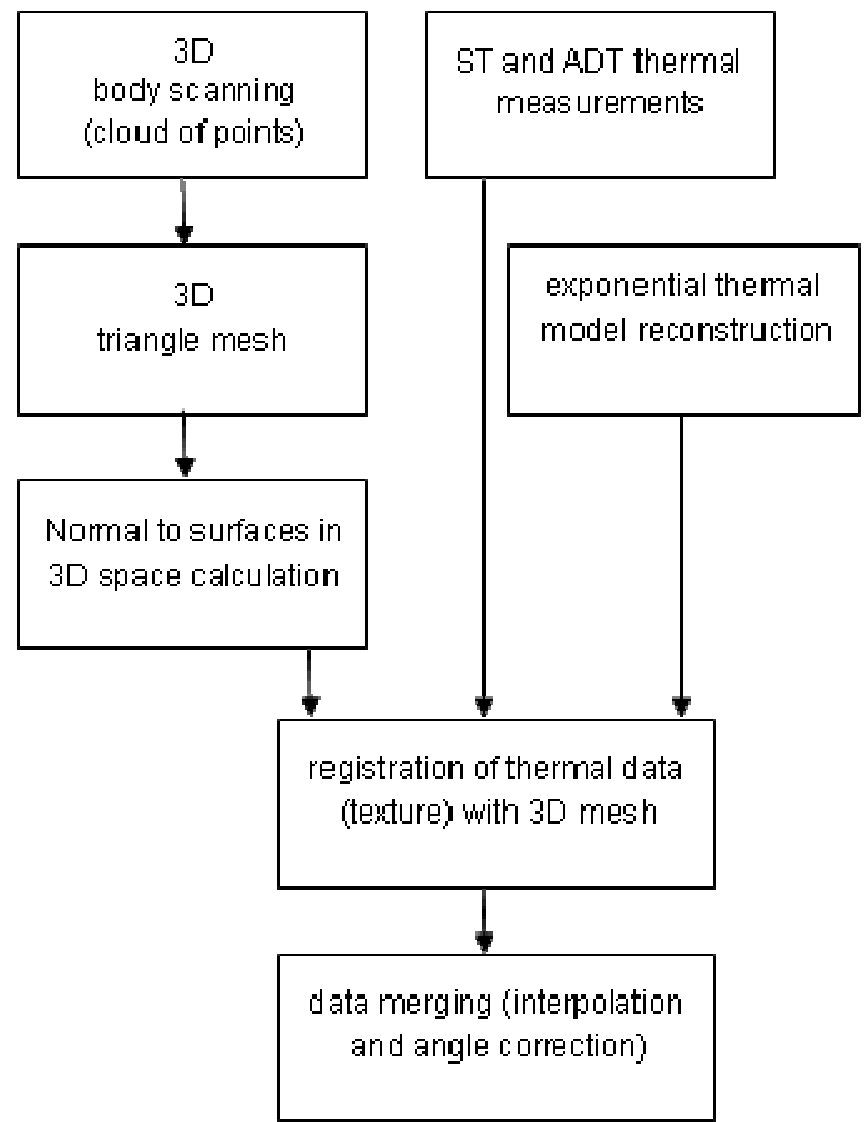

Fig. 3. Thermal data to3D model integration algorithm

The problems of technology development to properly perform the assumed tasks concern:

- Elaboration of the methodology combining multimodal images, including ADT, and extraction of diagnostically important features for new criteria of surgical wounds healing process estimation,

- Multimodal image matching, 3D model merging and diagnostic information presentation,

- Determination the influence of blood perfusion, mechanical stress, functional and morphological changes of the examined tissue on the resulting ADT imaging,

- Elaboration of the $3 \mathrm{D}$, thermal computer model of the examined structure for thermal processes simulation purposes and analysis basing on real measured 3D data, 
- $\quad$ Clinical experiments using all the important technologies to assembly statistically reliable diagnostic material in correlation with classical examinations confirmation.

- Checking the capabilities of using the elaborated methods and procedures in other surgical clinical applications, for example in the plastic surgery - breast reconstruction or blister forming diagnostic.

\section{Results}

As an example of obtained results the application of described methods for the purposes of the research project: evaluation of postoperative wound healing based on thermographic methods are shown. The individual patient examination lasts about 15 minutes during dressing change, utilizing each infrared technique as well as the physical and pathology methods. The 3D scanning process lasting no longer than 1 minute due to repeating scanning process for smaller parts of body and following merging algorithm. Data are stored as 3D mesh model. The aim of presented study is to obtain the reliable statistical insight for the procedure and the method validation for healing surgery wound process. The research also includes analysis of different conditions that may influence the process of wound healing. These risk factors are: age, gender, wound size, application of different healing alternating methods (such as the therapy with oxygen), complications etc. Acquired data are transferred to the database and analyzed by the dedicated software. As an example of measurements typical images of a chosen patient are presented in Fig. 4. Diagnostic information is extracted by further image data processing and comparing in consecutive examinations.

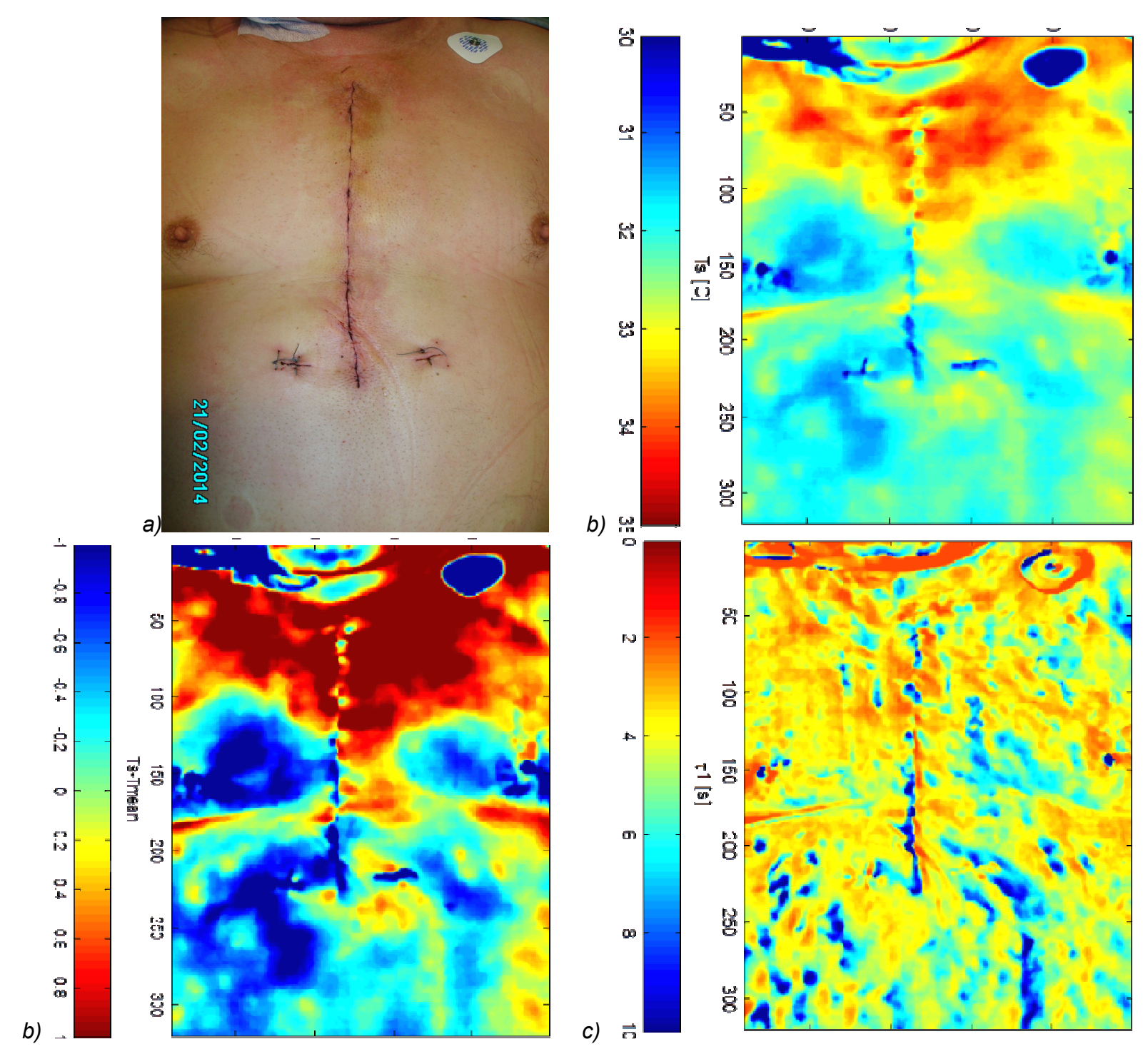



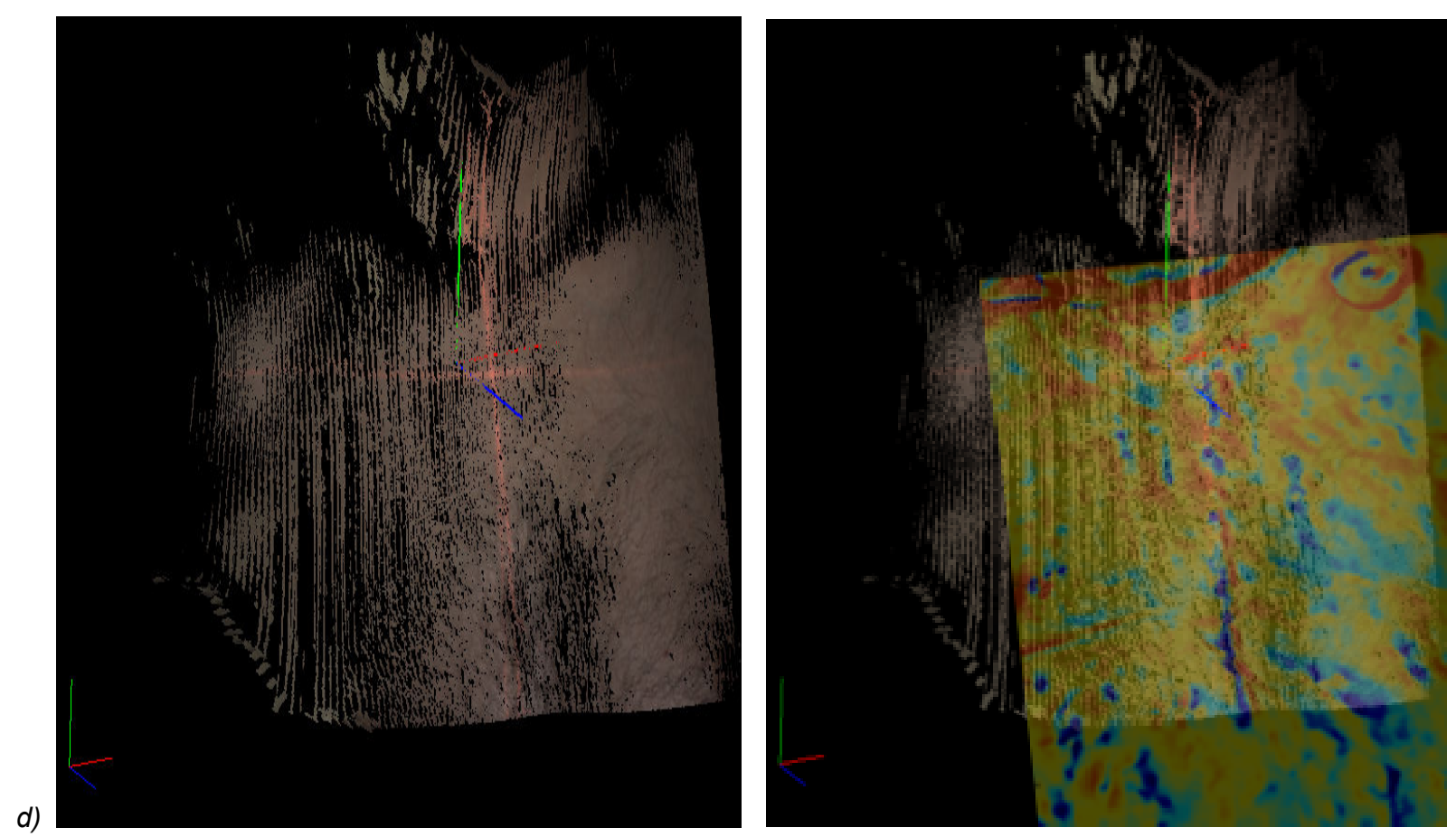

Fig. 4. Examples of medical application - wound healing application: a) photo, b) temperature distribution on static thermography (ST), c) parametric image of time constant in ADT procedure, d) $3 D$ mesh model and merged data

The project is a work in progress and over the next few months, we hope to have full scale results on data sets consisting of almost 300 patients with one thousand images and more. Shown above are some preliminary results of running our system.

\section{Conclusions}

The integration of structured light based 3D scanner and thermal camera enables acquisition and modelling of $3 \mathrm{D}$ thermogram data. 3D thermogram is a spatial model of observed object that contains surface shape and temperature distribution data. Thus it can be a valuable data model for current and future applications for modeling and simulating biological and physiological processes of the human body tissues. Presented technique could be very useful in e.g. face recognition systems [9] when we will be able to use in such systems real 3D models.

The white light 3D scanners have the main disadvantage: they are very sensitive to ambient light condition and have quite small measurement volume (e.g. in our case:300x300x400mm) what enforces the necessity of multiple measurements and combining multiple point clouds into a single model of thorax. Up to now the complicated procedure of reconstruction 3D triangle mesh from cloud of points is the most time consume routine. First of all, the artefacts according to bad ambient light condition (e.g. reflections) should be removed then next combining the points clouds into one model. There is generated the millions of points during the one scan, so triangle mesh generation is very sophisticated process.

So far, the process of thermal data and 3D model integration is the manual process. Currently we are working on automation of the system with a friendly and intuitive user interface. Automating the process is possible when you will be used tags clearly visible both in the visible and in the infrared range. It would be good if such markers could be glued throughout the patient's hospital stay (e.g. 7 days). Then you would be to able to register data, and to integrate and analyze data directly from various studies in automatic manner.

\section{Acknowledgement}

The work was financed by the Polish National Science Centre - the research grant NCN UMO2011/03/B/ST7/03423 „Opracowanie nowych deskryptorów obrazowania w podczerwieni dla obiektywnej oceny gojenia ran pooperacyjnych" Development of new IR-imaging descriptors for objective evaluation of post-operative wound healing, $2011-2014$.

\section{REFERENCES}

[1] Nowakowski A, Quantitative Active Dynamic Thermal IR-Imaging and Thermal Tomography in Medical Diagnostics, Diakides N, Bronzino J, editors. Medical Infrared Imaging, CRC Taylor\&Francis, 2008 : 7-1 - 7-29. 
[2] Ruminski J, Kaczmarek M, Renkielska A, et al. Thermal parametric imaging in the evaluation of skin burn depth. IEEE Trans. Biomed. Eng. 2007; 54(2): 303-312.

[3] Renkielska A, Nowakowski A, Kaczmarek M, et al. Burn depths evaluation based on active dynamic IR thermal imaging - a preliminary study. Burns 2006; 32: 867-875.

[4] Kaczmarek M., Nowakowski A., Suchowirski M., Siebert J., Stojek W., Active dynamic thermography in cardiosurgery, Quantitative InfraRed Thermography Journal. - Vol. 4, no 1 (2007), 107-123.

[5] http://www.smarttech3d.com/

[6] Rocchini C., Cignoni P., Montani C., Pingi P., Scopigno R., A low cost 3D scanner based on structured light, EUROGRAPHICS, 2001, Vol. 20, Num 3.

[7] Grubisic, I.; Gjenero, L.; Lipic, T.; Sovic, I.; Skala, T., Active 3D scanning based 3D thermography system and medical applications, MIPRO, 2011 Proceedings of the 34th International Convention, Issue Date: 23-27 May 2011.

[8] Ardeshir Goshtasby A., 2-D and 3-D Image Registration for Medical, Remote Sensing, and Industrial Applications, Wiley Press, 2005.

[9] Bauer J., Mazurkiewicz J., Neural Network and Optical Correlators for Infrared Imaging Based Face Recognition, Conference: Intelligent Systems Design and Applications - ISDA , pp. 234-238, 2005 